\title{
The Role of Mercury in Cardiovascular Disease
}

\section{Mark C. Houston ${ }^{1,2,3 *}$}

${ }^{1}$ Associate Clinical Professor of Medicine, Vanderbilt University School of Medicine, USA

${ }^{2}$ Director, Hypertension Institute and Vascular Biology, USA

${ }^{3}$ Medical Director, Division of Human Nutrition, Saint Thomas Medical Group, Saint Thomas Hospital, Nashville, Tennessee, USA

\begin{abstract}
Mercury toxicity is highly correlated with hypertension, coronary heart disease (CHD), myocardial infarction (MI), stroke and other cardiovascular disease. Mercury has a high affinity for sulfhydryl (-SH) groups, which inactivates numerous enzymatic reactions, amino acids, and sulfur-containing antioxidants [NAC ( $n$-acetyl cysteine, ALA (alpha lipoic acid), GSH (glutathione)], with subsequent decreased oxidant defense and increased oxidative stress. Mercury binds to metallothionein and substitutes for zinc, copper, and other trace metals reducing the effectiveness of metalloenzymes. Mercury induces mitochondrial dysfunction with reduction in ATP, depletion of glutathione, and increased lipid peroxidation. Selenium and fish containing omega 3 fatty acids or omega 3 fatty acids supplements with DHA and EPA antagonize mercury toxicity. The overall vascular effects of mercury include increased oxidative stress, inflammation, reduced oxidative defense, thrombosis, vascular smooth muscle dysfunction and hypertrophy, endothelial dysfunction, dyslipidemia, immune and mitochondrial dysfunction. The clinical consequences of mercury toxicity include hypertension, CHD, MI, cardiac arrhythmias, reduced heart rate variability, increased carotid IMT and carotid artery obstruction, CVA, generalized atherosclerosis, renal dysfunction, renal insufficiency and proteinuria. Pathological and biochemical findings correlate with the clinical manifestations of mercury toxicity. Mercury diminishes the protective effect of fish and omega-3 fatty acids. Mercury inactivates COMT (catecholamine 0 methyl transferase), which increases serum and urinary epinephrine, norepinephrine, and dopamine. This effect will increase blood pressure and may be a clinical clue to mercury- induced heavy metal toxicity. Mercury toxicity should be evaluated in any patient with hypertension, CHD, MI, CVD, CVA or other vascular disease. Specific testing for acute and chronic toxicity and total body burden using hair, toenail, urine and serum should be done with both baseline and provocation methods.
\end{abstract}

Keywords: Mercury toxicity; Hypertension; Cardiovascular disease; Coronary heart disease; Stroke; Renal disease; Oxidative stress; Inflammation

\section{Introduction}

Cardiovascular disease (CVD) is the leading cause of death worldwide. The overall health effects of chronic exposure to various heavy metals in the environment is a serious issue that has not received proper medical attention, especially as it relates to cardiovascular disease. This is particularly true of mercury but also with other heavy metals such as cadmium, lead, aluminum, iron and arsenic [1-5]. The cardiovascular consequences of mercury toxicity and the correlation of environmental pollution and the development of CVD have been well documented in the literature [1-80]. CERLA (The Comprehensive Environment Response, Compensation and Liability Act has prepared a list of substances that are most commonly pose the most significant potential threat to human health due to their known or suspected toxicity and potential for human exposure [6-8]. These include arsenic, lead and mercury in the top three. This paper will critically review the cardiovascular consequences of mercury toxicity in humans as it relates to hypertension, generalized atherosclerosis, coronary heart disease (CHD), myocardial infarction (MI), cardiac arrhythmias, heart rate variability (HRV), sudden cardiac death (SCD), cerebrovascular accidents (CVA), carotid artery disease, renal dysfunction, renal failure and total mortality.

\section{Types of mercury}

Mercury exists in three basic forms: elemental, inorganic and organic (Table 1) [1-5]. Dental amalgams are the most common source for elemental mercury vapor, a stable monoatomic gas. Inorganic mercury, a divalent compound, is the toxic species found in human tissue after conversion from the other forms. Organic mercury in the form of methyl and ethyl mercury is primarily from fish, sea mammals and thimersol vaccines. Although dental amalgams have historically been the major source of human exposure, fish and sea mammals are becoming an increasing environment source of potential mercury toxicity $[1,2,4,5]$.

\section{Mercury biotransformation and biomethylation}

Mercury exposure is from numerous sources. These include elemental mercury from earth sources or inhaled mercury vapor, methyl and ethyl mercury which are converted by biomethylation

\begin{tabular}{|l|l|l|l|}
\hline \multirow{2}{*}{1} & Elemental & Mercury Vapor $\left(\mathrm{Hg}^{\circ}\right)$ & Dental Amalgams \\
\cline { 3 - 4 } & Stable Monoatomic Gas & $\begin{array}{l}\text { Toxic species in human tissue } \\
\text { after conversion }\end{array}$ \\
\hline \multirow{2}{*}{3} & Inorganic & Divalent Mercury $\left(\mathrm{Hg}^{2+}\right)$ & Fish, sea mammals \\
\hline \multirow{2}{*}{\begin{tabular}{l} 
Organic \\
\cline { 2 - 3 }
\end{tabular}} & \begin{tabular}{l} 
Methyl Mercury $\left(\mathrm{CH}_{3} \mathrm{Hg}^{+}\right)$ \\
\cline { 2 - 3 }
\end{tabular} & Ethyl Mercury $\left(\mathrm{CH}_{3} \mathrm{CH}_{3} \mathrm{Hg}^{+}\right)$ & Thimersol vaccines \\
\hline
\end{tabular}

Table 1: Mercury types.

*Corresponding author: Mark Houston, Associate Clinical Professor of Medicine, Vanderbilt University School of Medicine, Director, Hypertension Institute and Vascular Biology, Medical Director, Division of Human Nutrition, Saint Thomas Medical Group, Saint Thomas Hospital, 4230 Harding Road, Suite 400, Saint Thomas Medical Plaza, Nashville, TN 37205, USA, Tel: 615-297-2700; Fax: 615373-0302; E-mail: boohouston@comcast.net

Received July 15, 2014; Accepted August 27, 2014; Published September 03 2014

Citation: Houston MC (2014) The Role of Mercury in Cardiovascular Disease. J Cardiovasc Dis Diagn 2: 170. doi:10.4172/2329-9517.1000170

Copyright: ( 2014 Houston MC. This is an open-access article distributed under the terms of the Creative Commons Attribution License, which permits unrestricted use, distribution, and reproduction in any medium, provided the original author and source are credited. 
to inorganic divalent mercury, the toxic form in human organs and tissues (Figure 1) [4]. Divalent mercury is soluble and stable in water, undergoes biomethylation to methyl mercury, which is found in high concentrations in certain fish and sea mammals. This is now the major source of human exposure to mercury.

The Environmental Protection Agency (EPA) has proposed that the safe daily intake of mercury is less than 0.1 microgram $/ \mathrm{kg} /$ day [6]. However any amount of mercury may have health consequences. Also, $12 \%$ of women have hair mercury above the level at which stopping consumption of highly contaminated fish would be advisable (1.0 $\mathrm{ug} / \mathrm{g}$ ) [7]. It is estimated that one dental amalgam filling releases about 3-17 micrograms of mercury vapor per day. The typical amalgam is composed of 50\% mercury, $25 \%$ silver and $25 \%$ tin, copper and nickels [7-10].

Fish and sea mammals provide about 2-3 micrograms per day depending on the type and amount consumed $[1,2,4,5]$. The long-lived large predatory fish such as swordfish, tilefish, shark and king mackerel contain about one microgram of methyl mercury per gram and should probably be avoided in the human diet. Pike, whale, bass, tuna and trout are about $0.1-0.5$ micrograms of mercury per gram. Nine vaccines that contain thimerosol (50\% mercury) as a preservative would give an estimated exposure of 62 micrograms of organic mercury [1,2,4,5]. All other sources of mercury provide about 0.3 micrograms per day $[1,2,4,5]$.

\section{Important Facts about Mercury}

Mercury is the most dangerous of all the heavy metals [11]. It will modify the distribution, toxicity and retention of other heavy metals [12-14]. Mercury has no known physiological role in human metabolism and there are no mechanisms to metabolize or to excrete mercury acutely or chronically [15]. Mercury will accumulate during life. The average $70 \mathrm{~kg}$ person has a total body burden of over $13 \mathrm{mg}$ of mercury [11]. Mercury has a high affinity for sulfhydryl groups (-SH), various enzymes and amino acids, alpha lipoic acid (ALA), N-acetyl cysteine (NAC), and glutathione (GSH), which provide between 10-

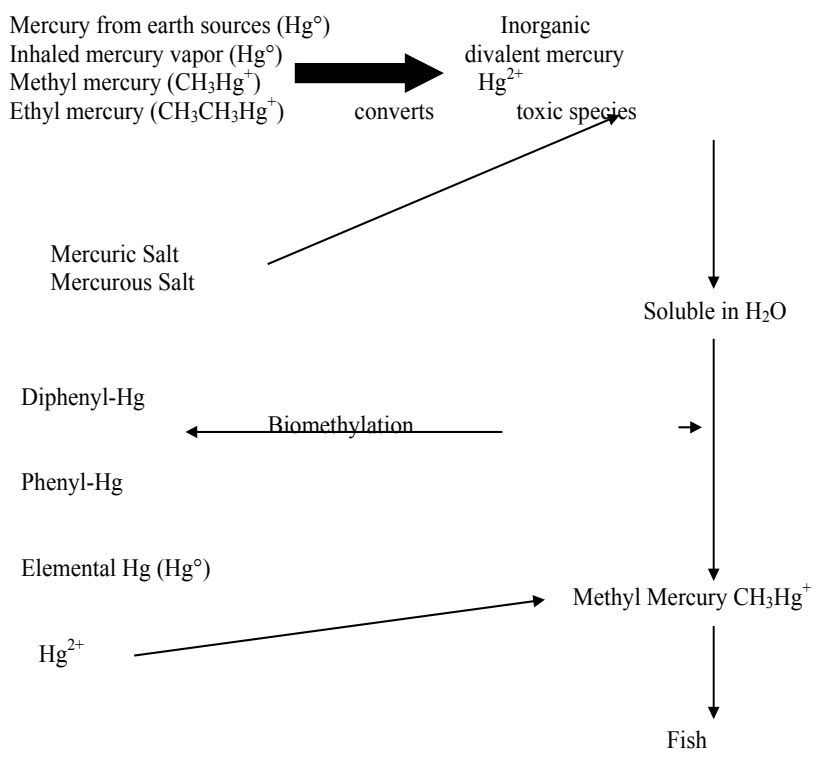

Figure 1: Mercury biotransformation and biomethylation.
$50 \%$ of the plasma protein antioxidant defense and capacity $[11,15,16]$. Both NAC and ALA, as well as cysteine, are precursors for glutathione, which is the most potent intracellular anti-oxidant and protects against oxidative stress, inflammation, immune dysfunction, infections, carcinoma and cardiovascular disease $[3-5,11,12,15]$. This mercuryinduced reduction in oxidant defense and increase in oxidative stress increase the risk for CHD, MI, CVD and CVA. Selenium antagonizes some of the adverse effects of mercury by forming a seleno-mercury complex in tissue that is less toxic [12,18-24]. Higher intake of selenium reduces mercury related CHD, MI, CVD and CVA. Selenium in supplemental form should not exceed 200 micrograms per day.

\section{Physiological basis of mercury toxicity}

Mercury induces mitochondrial dysfunction, decreases ATP production and energy and increases oxidative stress [25-27]. The primary mitochondrial dysfunction occurs at the ubiquinonecytochrome $\mathrm{B}$ region and with $\mathrm{NADH}$ dehydrogenase causing displacement of $\mathrm{Fe}^{2+}$ and $\mathrm{Cu}^{+}$ions in the a3Cub center of cytochrome $\mathrm{C}$ (Figure 2). The depolarization and auto-oxidation of the inner mitochondrial membrane with lipid peroxidation lead to severe mitochondrial dysfunction. Physiologic consequences include increased hydrogen peroxide $\left(\mathrm{H}_{2} \mathrm{O}_{2}\right)$, depletion of mitochondrial glutathione by $50 \%$ or more, increased lipid peroxidation markers such as TBARS by over $70 \%$, oxidation of pyridine nucleotides such as $\mathrm{NAD}(\mathrm{p}) \mathrm{H}$ and altered calcium homeostasis [25-27]. Increase in the intracellular calcium results in cell apoptosis and death. This severe mitochondrial dysfunction increases oxidant stress and reduces oxidant defenses that increase the risk of cardiovascular disease.

The three primary sources of mercury- induced lipid peroxidation include the affinity for sulfhydryl groups, selenium deficiency and the Fenton reaction [11]. Mercury's high affinity for sulfhydryl groups (-SH), such as glutathione, NAC and ALA, which comprise much of the antioxidant capacity of plasma, reduces both membrane and plasma antioxidant defense. Mercury is a direct catalyst in Fenton-type reactions and as an indirect catalyst via iron stimulation, which increases the production of radical oxygen species (ROS) and superoxide anion [11]. Insoluble complexes of mercury with selenium reduce selenium availability, which is a necessary co-factor for glutathione peroxidase (GPx) activity to break down hydrogen peroxides and various other toxic peroxidation products. A reduced GPx and low GSH levels are associated with increased risk for CHD and MI. Plasma and intracellular antioxidant capacity are both reduced as GSH levels fall [11].

\section{Vascular biological effects of mercury}

In vitro, animal and human studies have demonstrated numerous toxic effects of mercury (Table 2). Mercury increases free radical production and ROS and damages DNA inactivates antioxidant defenses and depletes vitamin $\mathrm{C}$ and $\mathrm{E}$, binds to thiol-containing molecules, binds to selenium forming seleno-mercury complexes lowering selenium availability for GPx activity inactivates glutathione, catalase and superoxide dismutase increases lipid peroxidation, increases oxidation of LDL (oxLDL) and increases plasma oxLDL complexes inducing an immune reaction [3,28-39]. Thrombosis is potentiated by increased platelet aggregation, change in platelet shape, abnormal erythrocyte shape and microvesicle generation increases in Factor VIII platelet factor- 4 and thrombin with reduction in protein $\mathrm{C}[28,40,41]$. Endothelial cell formation and migration are reduced from endothelial progenitor cells (EPCs) which reduce vascular endothelial repair; nitric oxide $(\mathrm{NO})$ bioavailability is decreased with subsequent endothelial dysfunction which is one of 


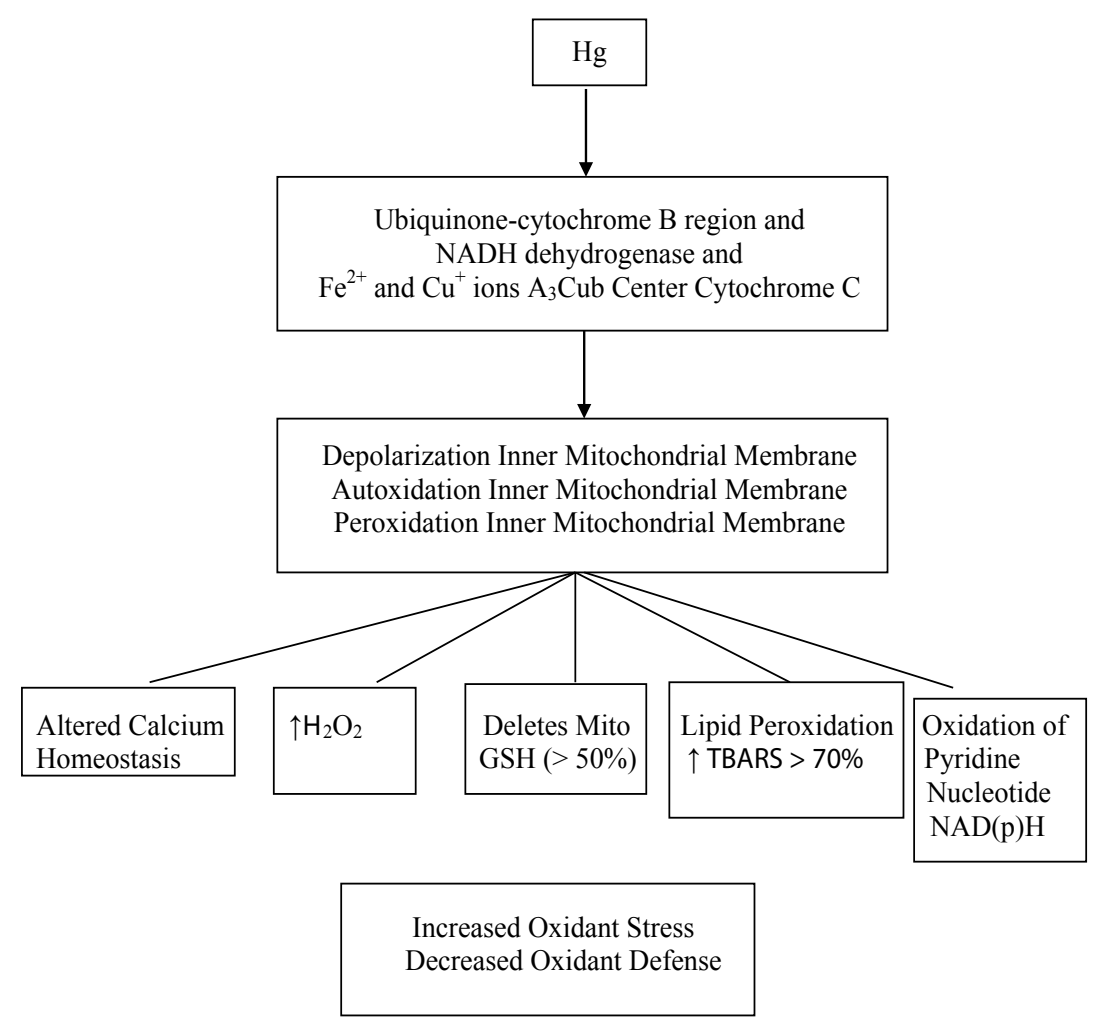

Figure 2: Pathophysiologic basis of mercury toxicity, mitochondrial dysfunction and oxidative stress.

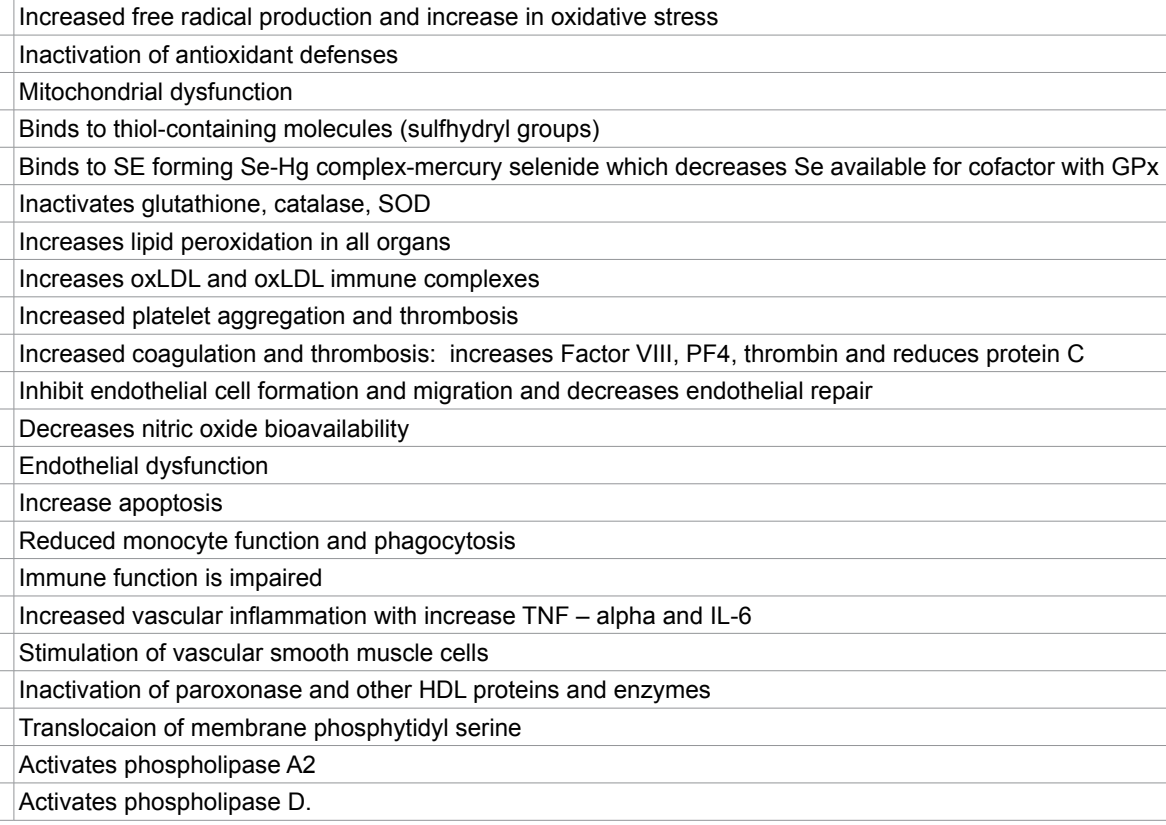

Table 2: Vascular biological effects of mercury.

the earliest predictors of future CV events [42]. Apoptosis is increased, monocyte function and phagocytosis are impaired immune function is reducedand vascular inflammation is increased with elevations of HS CRP (High sensitivity C reactive protein) TNF alpha (tumor necrosis alpha) and IL-6 (interleukin 6) [30]. There is an increased production and release of superoxide anion from human neutrophils and monocytes depolarization of the inner mitochondrial membrane with severe mitochondrial dysfunction and disruption of plasma membrane lipid integrity by translocation of phosphytidyl serine (PS) which will increase procoagulant activity [25-30]. Mercury stimulates proliferation of vascular smooth muscle (VSMC) cells and inactivates paraoxonase (PON 1 and 2), an extracellular antioxidative enzyme 
related to HDL, CHD and MI risk [43-45]. The reduction in PON will make the HDL dysfunctional, reduces reverse cholesterol transport and increase the risk of CHD, MI and CVD. The clinical consequences of these and other pathophysiologic mechanisms explains the wide variety of cardiovascular diseases caused by mercury including CHD, MI, arrhythmias abnormal heart rate variability (HRV), generalized atherosclerosis, sudden cardiac death, CVA, carotid artery stenosis, renal dysfunction, renal failure, proteinuria and hypertension. $[4,5,9,10-12,16,19,23,32,46-80]$. Mercury activates phospholipase A2 (PLA-2) which increases the risk for coronary artery and cerebral plaque rupture with $\mathrm{MI}$ and CVA. In addition, mercury induces formation of arachidonic acid metabolites such as total prostaglandins, thromboxane B2 and 8 isoprostane in vascular endothelial cells and activates vascular endothelial cell phospholipase D [59-63]. Even very low levels of chronic mercury exposure promote endothelial dysfunction (ED) as a result of increased inflammation, oxidative stress, immune dysfunction, reduced oxidative defense, reduction in nitric oxide (NO) bioavailability. Many of the cardiovascular consequences of mercury are mitigated by concomitant intake of fish containing omega 3 fatty acids and by the intake of selenium [11,53-58]. All of these pathobiological findings will increase the risk of hypertension, CHD, MI, CVD and CVA [27].

In summary, the overall vascular effects of mercury include oxidative stress, decreased oxidative defense, increased inflammation, immune dysfunction, VSM proliferation, hypertrophy and migration, endothelial dysfunction, reduced NO bioavailability, dyslipidemia and mitochondrial dysfunction and thrombosis. All of these abnormalities have the potential to increase the risk for hypertension, CHD, MI, CVD and CVA.

\section{Clinical vascular consequences of mercury toxicity}

The clinical consequences of mercury toxicity related to cardiovascular disease include hypertension, CHD, MI, reduction in heart rate variability increase in sudden cardiac death increase in carotid intimal medial thickness (IMT) and carotid obstruction, CVA, generalized atherosclerosis, renal dysfunction, renal failure and proteinuria4 and an overall increase in total and cardiovascular mortality $[4,5,16-19,23,32,46-49,19,48,64-70,73,80]$. In a study of Faroese whaling men, both toenail and hair mercury levels were significantly associated with increased carotid IMT and hypertension [78]. Gomez et al. followed 3,998 workers in mercury mines exposed to inorganic mercury from 1895 to 1994 and found a $2.78 \mathrm{x}$ increase incidence of hypertension, $1.17 \mathrm{x}$ increase risk of stroke and $1.51 \mathrm{x}$ increase risk in total cardiovascular mortality, but no increase in CHD [78]. Evidence from these and other epidemiologic and clinical studies suggest that people with high levels of urine, hair, blood and toenail mercury have an increased risk of cardiovascular diseases $[5,11,16,58,65,68,69,80]$.

\section{Coronary heart disease and myocardial infarction}

The cardiovascular and cardiac pathology in rabbits exposed to inhaled mercury vapor are clinically manifest as endothelial proliferation with inflammatory foci and focal edema, bradycardia, thrombosis in small and medium caliber arteries, papillary muscles and valves, focal necrosis with thickening of the endocardium of the perivalvular regions, inflammation and fibrosis of the ascending aorta [49].

A nine country case control study of 684 men with their first MI, demonstrate a significant association of toenail mercury content, adipose tissue DHA and first MI [5]. A 15\% higher toenail mercury content as assessed by neutron activation analysis (NAA) was found in the men with their first MI compared to the control group (95\% CI; 5-25\%). The OR, risk adjusted, for MI was 2.16 in highest versus the lowest quintile ( $\mathrm{p}=0.006,95 \% \mathrm{CI} ; 1.09-4.29)$. The adipose DHA was directly proportional to the mercury toenail content $(\mathrm{p}<0.001)$ and the DHA content was inversely correlated to MI with an OR of 0.59 in the highest versus the lowest quintile ( $\mathrm{p}=0.02,95 \%$ CI; $0.30-1.19$ ). They concluded that there exists a positive, monotonic increase in the risk of MI with mercury toenail content above the $0.25 \mathrm{microgram} / \mathrm{gram}$ level. This association was even more pronounced when adjusted for the DHA adipose tissue content. Mercury diminishes the cardiovascular protection of fish consumption and omega 3 fatty acids. Another study substantiated these results in which the highest quartile of DHA with the lowest quartile of mercury was associated with a $67 \%$ reduction in CHD $(p<0.016)$ [50]. In the Finish Kuopio Ischaemic Heart Disease Risk Factor Study a cohort of 1871 Finnish men age 42-60 years free of previous coronary heart disease or stroke at baseline found a direct relationship of mercury hair content and CVD, CHD and all-cause mortality. Mercury also attenuated the protective effects of fish on cardiovascular health [51].

However, in another large nested case control study of 33,733 male healthcare professionals between the ages of 40-75 years (Health Professionals Follow-Up Study, HCPFUS), no association between mercury toenail content assessed by NAA and CHD was found [12]. However, if dentists were included, there was a nonsignificant correlation of toenail mercury and CHD. Also, subjects with the highest tertile of mercury and the lowest serum selenium level had a significant increase in CHD.

Other human studies have shown mixed results $[9,11,46,47,52]$. Mercury miners had no relationship between CHD and serum mercury levels [32]. However, another study of European mercury miners over 90 years (6784 males and 265 females from Spain, Sloevenia, Italy and the Ukraine) showed a significant relationship of mercury exposure to total mortality (increase 8\%), CHD (increase 36\%), myocarditis, hypertension (increase 46\%), renal disease (increase 55\%) and CVA (increase 36\%) [46]. The incidence of CVD correlated with the time of onset of the first exposure to mercury. A Finnish study found a significant relationship between hair mercury, 24-hour urine mercury and cardiovascular events [11]. In patients with hair mercury in the highest tertile, over 2.0 micrograms/gram, and increased 24-hour urinary mercury, CHD and MI risk were increased two-fold $(\mathrm{p}=0.005)$, cardiovascular death increased by 2.9 times $(\mathrm{p}=0.014)$ and circulating oxLDL and immune complexes to oxLDL increased significantly $\mathrm{p}=0.01$ ). The National Health and Nutrition Examination Survey from 1999-2002 found levels of DHA and EPA and other nutrients in fish, even with elevated mercury levels, helped to offset the risk of CHD and MI [71]. The fish intake resulted in lower levels of CRP and higher serum HDL cholesterol as well [71]. The Gothenburg Study showed no relationship between serum mercury content and the number of amalgam fillings and CHD or MI [9]. Exposure to methylmercury in a Finnish and Swedish population was associated with increased risk of MI and higher PUFA concentrations were associated with a decreased risk of MI [72]. In this study, hair mercury had a significant correlation with $\mathrm{MI}$ at $\mathrm{p}=0.005)$. A change of 1 percent omega 3 fatty acids in fish consumption was associated with a 7\% reduction in MI. High exposure to mercury reduced the benefits of long chain n-3 PUFA in the 20 year follow-up of the Kuopio Heart Disease Risk Factor Study of 1857 men [73]. For each .5 percentage unit increase in serum long-chain n-3 PUFA was associated with HR of .77 for sudden cardiac death, based on hair mercury content [73]. The DHA level was most strongly 
correlated. In a US cohort of 51,529 men and 121,700 women, there was no association of toenail mercury content (neutron activation analysis, NAA) and risk of CHD, CVA or total CVD in the 3427 participants that were prospectively studied [74]. The toenail mercury content was $.23 \mathrm{ug} / \mathrm{gram}$ in the case participants and $.25 \mathrm{ug} / \mathrm{gram}$ in the controls. A study of 274 children from South Korea found a significant correlation of urine mercury levels and serum cholesterol which would increase the risk of CHD [75].

\section{Stroke and carotid atherosclerosis}

High hair mercury content increases carotid IMT and carotid atherosclerosis [16]. An increase in mean carotid IMT during a four year follow-up was related directly to hair mercury content ( $\mathrm{p}=0.0007$ ), in a study of 1,014 men between the ages of 42-60 years [16]. Each increase of one microgram in hair mercury content correlated with a $0.008 \mathrm{~mm}$ increase in carotid IMT, a 7.3\% increase over the mean. There was a $0.042 \mathrm{~mm} / 4$ years increase in carotid IMT in the highest quintile versus the lowest quintile, which correlated to a $32 \%$ greater increase $(\mathrm{p}<0.05)$. In addition, mercury hair content was proportional to blood pressure levels, waist hip ratio, fibrinogen and low HDL cholesterol; all significant at $\mathrm{p}=0.0002$. Other studies have shown a direct relationship between mercury vapor exposure and urinary mercury and early increase in carotid IMT, asymptomatic carotid atherosclerosis, and low HDL cholesterol and reduced total antioxidant capacity [17]. Many studies on the risk of fish intake, mercury and stroke have been inconclusive. In addition, the various types of stroke have often not been separated or well defined. In a population-based cohort, mercury levels and relative content of fatty acids were determined in erythrocyte membranes in the population consuming one meal per week as fish [77]. In women, there was a non-significant decrease in stroke risk with increasing fish intake with OR of .90. The risk in men for stroke rose with increasing fish intake with OR of 1.24. The corresponding risk in men for mercury was 0.99 and for the sum of proportions of EPA and DHA was 1.08 . This study suggested that the risk for stroke between genders differs with increasing fish intake, EPA and DHA consumption, but there was no association between stroke risk and mercury at these lower levels of one meal of fish per week. There are many basic mechanisms by which mercury can increase the risk for stroke as discussed earlier in this paper. The increases in blood pressure, pulse pressure, increased augmentation index, arterial stiffness increased thrombotic risk related to increased platelet aggregation, increase in Factor VIII, thrombin and platelet factor 4 and reduction in protein $\mathrm{C}$ as well as endothelial dysfunction from reduced $\mathrm{NO}$ bioavailability may account for much of the observed elevation in CVA risk with mercury [16,40$42,46,47,52,64-66,76,68-80]$. One recent study showed that mercury increases thrombotic risk by enhancement of procoagulant activity in erythrocytes by protein thiol depletion-mediated phosphatidyl serine exposure and microvesical generation [78].

\section{Hypertension}

The association of mercury toxicity and hypertension in humans is well documented and convincing $[16,32,46,47,52,64-80]$. Mercury miners have significant increases in systolic blood pressure $(\mathrm{p}<0.01)$ that correlated with oxidative stress, reduced oxidative defense and lipid peroxidation $(p<0.01)$ [32]. European mercury miners had a $46 \%$ greater incidence of hypertension vs. aged-matched controls. The Wisconsin Sleep Cohort study of over 100 subjects found that those in the upper quartile of blood mercury were 1.9 times more likely to be hypertensive ( $p=0.023$ ) [66]. The subjects in the upper quartile of hair mercury were 4 times more likely to be hypertensive than those in the lowest quartile $(\mathrm{p}=0.02)$. There was no change in brachial artery flow mediated vasodilation or the middle cerebral artery reactivity to $\mathrm{CO}_{2}$ [66]. Other studies have shown significant correlations with hair mercury content, hypertension, diastolic dysfunction, $\mathrm{LVH}$ and carotid IMT [16]. Over 250 subjects in the Brazilian Amazon were found to have significant increases in BP associated with total hair mercury levels. The OR for elevated SBP with total hair mercury over $10 \mathrm{mcg} / \mathrm{g}$ was 2.91 (1.26-7.28) [65]. Inuit adults had blood mercury levels that were correlated with pulse pressure $(\mathrm{p}=0.0004)$, SBP $(\mathrm{p}<0.0001)$ and $\mathrm{DBP}(\mathrm{p}=0.069)$ [67]. In a comparative population study of 833 subjects, long term methyl mercury exposure, as measured by hair mercury levels, was associated with a risk of hypertension of 1.4 to 1.6 times in [68]. In a sample of 1240 women age 16-49 who participated in the National Health and Nutrition Examination Survey 1999-2000 a significant increase in SBP with increasing levels of blood total mercury was demonstrated, but only among non-fish consumers [69]. There was a $1.83 \mathrm{~mm} \mathrm{Hg}$ increase in SBP for each $1.3 \mathrm{ug} / \mathrm{L}$ increase in blood total mercury ( $95 \%$ CI: $0.36,3.30$; interaction $\mathrm{p}=0.02$ ). The risk of hypertension over ten years was highly correlated in a group of chemical factory workers exposed to mercury vapor [76].

Pederson et al. [77] found an increase in pulse pressure using 24 hour ambulatory blood pressure monitoring $(54 \mathrm{~mm} \mathrm{Hg}$ vs $50 \mathrm{~mm} \mathrm{Hg}$ with $\mathrm{p}<0.0001$ ) that was related to blood mercury levels ( $\mathrm{rho}=0.272$, $\mathrm{p}<0.01)$ in a group of Greenlanders consuming more fish than a group of Danes. Mercury is also significantly associated with reduced heart rate variability (HRV) in addition to the increased pulse pressure and hypertension [77]. A reduced HRV may predispose to ventricular fibrillation and sudden cardiac death, as well as being associated with angina, MI, CHD, CHF and all-cause mortality [77].

In acute and chronic mercury intoxication, mercury binds to the sulfhydryl group S-adenosyl methionine (SAM) and inactivates this enzyme, which is a necessary cofactor for catecholaminei-0-methyl transferase (COMT), the enzyme needed to metabolize norepinephrine, epinephrine and dopamine by methoxylation [47]. This results in a clinical syndrome that resembles a pheochromocytoma crisis with malignant hypertension in acute mercury intoxication and significant increases in urinary catecholamines in chronic mercury toxicity. This can be a very helpful clinical clue to mercury-induced hypertension. It would be important to measure baseline and provoked 24 hour urine mercury levels in patients with hypertension and a history or evidence clinically of possible mercury exposure. Mercury also induces renal dysfunction and proteinuria, which contribute to sodium retention and hypertension $[31,46,47,53]$. In such cases, it is important to evaluate both potassium and magnesium balance, as hyperkalemia and hyper-magnesemia are common metabolic problems with renal dysfunction. This is especially common with renal tubular disease (renal tubular acidosis-RTA) and the inability to adequately secrete potassium. As the GFR drops below about $20 \mathrm{cc}$ per minute the risk of hyperkalemia increases. Studies have shown an increase in renal insufficiency in mercury miners by 55\% [46]. Mercury concentrates in the renal tubules and in the glomerulus and results in proteinuria, interstitial and glomerular fibrosis, chronic renal dysfunction and renal insufficiency $[31,52]$.

The most effective means to reduce mercury toxicity is to decrease the mercury load from the offending source and treat with either oral or intravenous chelation therapy. Chelation therapy is beyond the scope of this paper but the reader is referred to the prolific literature on this topic (Table 3). 


\begin{tabular}{|l|l|}
\hline 1 & Oxidative Stress \\
\hline 2 & Inflammation \\
\hline 3 & Thrombosis \\
\hline 4 & Vascular Smooth Muscle (VSM) Proliferation and Migration \\
\hline 5 & Endothelial Dysfunction \\
\hline 6 & Dyslipidemia (oxHDL and paraxonase \\
\hline 7 & Immune Dysfunction \\
\hline 8 & Mitochrondrial Dsfunction \\
\hline
\end{tabular}

Mitochrondrial Dsfunction

Table 3: Summary of the overall vascular biologic effects of mercury.

\section{Summary}

1. Mercury has a high affinity for sulfydryl(-SH) groups, which inactivate numerous enzymatic reactions, amino acids and sulfur-containing antioxidants (NAC, ALA, GSH) with decreased oxidant defense and increased oxidative stress. Mercury binds to metallothionein and substitutes for zinc, copper and other trace metals reducing the effectiveness of metalloenzymes.

2. Mercury induces mitochondrial dysfunction, lowers ATP production, depletes glutathione and increases lipid peroxidation.

3. Selenium and fish high in omega 3 fatty acid content antagonize mercury toxicity.

4. The overall vascular effects of mercury include increases in oxidative stress, immune dysfunction and inflammation, reduction in oxidative defense, vascular smooth muscle dysfunction, endothelial dysfunction, dyslipidemia, thrombosis, and mitochondrial dysfunction.

5. The clinical consequences of mercury toxicity include hypertension, CHD, MI, cardiac arrhythmias, LVH, diastolic dysfunction, sudden cardiac death, reduced HRV, increased carotid IMT and carotid artery obstruction, CVA, generalized atherosclerosis and renal dysfunction, renal failure, insufficiency and proteinuria. Pathological and biochemical findings correlate significantly with the clinical CV manifestations.

6. Mercury diminishes the protective effect of fish and omega 3 fatty acids.

7. Mercury inactivates COMT (catecholamine-0-methyl transferase), which increases serum and urinary epinephrine, norepinephrine and dopamine. This effect will increase blood pressure and may be a clinical clue to mercury toxicity.

8. Mercury toxicity should be evaluated in any patient with hypertension, CVD, CHD, CVA or other vascular disease that have a clinical history of exposure or clinical evidence on examination of mercury overload. Specific testing for acute and chronic toxicity and total body burden using hair, toenail, urine and serum should be done. The 24 hour urine measurements should be done with baseline and provoked samples.

\section{References}

1. Keating MH, Mahaffey KR, Schoemy R, Rice GE, Bullock R, et al. (1997) Mercury study report to Congress. Vol. I. Executive summary. EPA452/R-97-003, Environmental Protection Agency, Washington, DC.

2. (2000) Committee on the Toxicological Effects of Methylmercury, Board on Environmental Studies and Toxicology, Commission on Life Sciences. Toxicological effects of methylmercury. National Research Council, Washington, DC.

3. Magos L (1997) Physiology and toxicology of mercury. Met lons Biol Syst 34: 321-370.

4. Clarkson TW, Magos L, Myers GJ (2003) The toxicology of mercury-current exposures and clinical manifestations. N Engl J Med 349: 1731-1737.
5. Guallar E, Sanz-Gallardo MI, van't Veer P, Bode P, Aro A, et al. (2002) Mercury fish oils, and the risk of myocardial infarction. N Engl J Med 347: 1747-1754.

6. Weinhold B (2004) Environmental cardiology: getting to the heart of the matter. Environ Health Perspect 112: A880-887.

7. Bhatnagar A (2006) Environmental cardiology: studying mechanistic links between pollution and heart disease. Circ Res 99: 692-705.

8. (2012) CERCLA Priority List of Hazardous Substances.

9. Ahlqwist M, Bengtsson C, Lapidus L, Gergdahl IA, Schütz A (1999) Serum mercury concentration in relation to survival, symptoms, and diseases: results from the prospective population study of women in Gothenburg, Sweden. Acta Odontol Scand 57: 168-174.

10. Bergdahl IA, Schütz A, Ahlqwist M, Bengtsson C, Lapidus L, et al. (1998) Methylmercury and inorganic mercury in serum--correlation to fish consumption and dental amalgam in a cohort of women born in 1922. Environ Res 77: 20-24

11. Salonen JT, Seppänen K, Nyyssönen K, Korpela H, Kauhanen J, et al. (1995) Intake of mercury from fish, lipid peroxidation, and the risk of myocardial infarction and coronary, cardiovascular, and any death in eastern Finnish men. Circulation 91: 645-655.

12. Yoshizawa K, Rimm EB, Morris JS, Spate VL, Hsieh CC, et al. (2002) Mercury and the risk of coronary heart disease in men. N Engl J Med 347: 1755-1760.

13. Chmielnicka J, Bem EM, Kaszubski P (1983) Organ and subcellular distribution of cadmium in rats exposed to cadmium, mercury, and selenium. Environ Res 31: $266-272$.

14. Komsta-Szumska E, Chmielnicka J (1983) Effect of zinc, cadmium or copper on mercury distribution in rat tissues. Toxicol Lett 17: 349-354.

15. International Programme on Chemical Safety (IPCS) (1990) Methylmercury. Environmental Health Criteria 101. Geneva: World Health Organization, USA.

16. Salonen JT, Seppänen K, Lakka TA, Salonen R, Kaplan GA (2000) Mercury accumulation and accelerated progression of carotid atherosclerosis: a population-based prospective 4-year follow-up study in men in eastern Finland Atherosclerosis 148: 265-273.

17. Skoczyska A, Poreba R, Steinmentz-Beck A, Martynowicz H, Affelska-Jercha A, et al. (2009) The dependence between urinary mercury concentration and carotid arterial intima-media thickness in workers occupationally exposed to mercury vapour. Int J Occup Med Environ Health 22: 135-142.

18. Parízek J, Ostádalová I (1967) The protective effect of small amounts of selenite in sublimate intoxication. Experientia 23: 142-143.

19. Ganther HE, Goudie C, Sunde ML, Kopecky MJ, Wagner P (1972) Selenium relation to decreased toxicity of methylmercury added to diets containing tuna Science 175: 1122-1124.

20. Ganther HE, Sunde ML (1974) Effect of tuna fish and selenium on the toxicity of methylmercury: a progress report. J Food Sci 39:1-5.

21. Stoewsand GS, Bache CA, Lisk DJ (1974) Dietary selenium protection of methylmercury intoxication of Japanese quail. Bull Environ Contam Toxicol 11: 152-156.

22. Sumino K, Yamamoto R, Kitamura S (1977) A role of selenium against methylmercury toxicity. Nature 268: 73-74.

23. Singhal RK, Anderson ME, Meister A (1987) Glutathione, a first line of defense against cadmium toxicity. FASEB J 1: 220-223.

24. Seppänen K, Kantola M, Laatikainen R, Nyyssönen K, Valkonen VP, et al (2000) Effect of supplementation with organic selenium on mercury status as measured by mercury in pubic hair. J Trace Elem Med Biol 14: 84-87.

25. Lund BO, Miller DM, Woods JS (1993) Studies on Hg (II)-induced H2O2 formation and oxidative stress in vivo and in vitro in rat kidney mitochondria. Biochem Pharmacol 45: 2017-2024.

26. Shenker BJ, Guo TL, Shapiro IM (1998) Low-level methylmercury exposure causes human T-cells to undergo apoptosis: evidence of mitochondrial dysfunction. Environ Res 77: 149-159.

27. Jansson G, Harms-Ringdahl M (1993) Stimulating effects of mercuric- and silver ions on the superoxide anion production in human polymorphonuclear leukocytes. Free Radic Res Commun 18: 87-98. 
28. Clarkson TW (1997) The toxicology of mercury. Crit Rev Clin Lab Sci 34: 369-403.

29. Insug O, Datar S, Koch CJ, Shapiro IM, Shenker BJ (1997) Mercuric compounds inhibit human monocyte function by inducing apoptosis: evidence for formation of reactive oxygen species, development of mitochondrial membrane permeability transition and loss of reductive reserve. Toxicology 124: 211-224.

30. Wiggers GA, Pecanha FM, Briones AM, Perez-Giron JV, Miguel M, et al. (2008) Low mercury concentrations cause oxidative stress and endothelial dysfunction in conductance and resistance arteries. Am J Physiol Heart Circ Physiol 295: 1033-1043.

31. Kobal AB, Horvat M, Prezelj M, Briski AS, Krsnik M, et al. (2004) The impact of long-term past exposure to elemental mercury on antioxidative capacity and lipid peroxidation in mercury miners. J Trace Elem Med Biol 17: 261-274.

32. Park ST, Lim KT, Chung YT, Kim SU (1996) Methylmercury-induced neurotoxicity in cerebral neuron culture is blocked by antioxidants and NMDA receptor antagonists. Neurotoxicology 17: 37-45.

33. Miller DM, Lund BO, Woods JS (1991) Reactivity of $\mathrm{Hg}(\mathrm{II})$ with superoxide: evidence for the catalytic dismutation of superoxide by $\mathrm{Hg}(\mathrm{II})$. J Biochem Toxicol 6: 293-298.

34. Naganuma A, Koyama Y, Imura N (1980) Behavior of methylmercury in mammalian erythrocytes. Toxicol Appl Pharmacol 54: 405-410.

35. Cuvin-Aralar ML, Furness RW (1991) Mercury and selenium interaction: a review. Ecotoxicol Environ Saf 21: 348-364.

36. Rungby J, Ernst E (1992) Experimentally induced lipid peroxidation after exposure to chromium, mercury or silver: interactions with carbon tetrachloride. Pharmacol Toxicol 70: 205-207.

37. Lin TH, Huang YL, Huang SF (1996) Lipid peroxidation in liver of rats administrated with methyl mercuric chloride. Biol Trace Elem Res 54: 33-41.

38. Wierzbicki R, Prazanowski M, Michalska M, Krajewska U, Mielicki WP (2002) Disorders in blood coagulation in humans occupationally exposed to mercuric vapors. J Trace Elem Exp Med 15: 21-29.

39. Kishimoto T, Oguri T, Abe M, Kajitani H, Tada M (1995) Inhibitory effect of methylmercury on migration and tube formation by cultured human vascular endothelial cells. Arch Toxicol 69: 357-361.

40. Lu KP, Zhao SH, Wang DS (1990) The stimulatory effect of heavy metal cations on proliferation of aortic smooth muscle cells. Sci China B 33: 303-310.

41. Gonzalvo MC, Gil F, Hernández AF, Villanueva E, Pla A (1997) Inhibition of paraoxonase activity in human liver microsomes by exposure to EDTA, metals and mercurials. Chem Biol Interact 105: 169-179.

42. Salonen JT, Malin R, Tuomainen TP, Nyyssönen K, Lakka TA, et al. (1999) Polymorphism in high density lipoprotein paraoxonase gene and risk of acute myocardial infarction in men: prospective nested case-control study. BMJ 319: 487-489.

43. Boffetta P, Sällsten G, Garcia-Gómez M, Pompe-Kirn V, Zaridze D, et al. (2001) Mortality from cardiovascular diseases and exposure to inorganic mercury. Occup Environ Med 58: 461-466.

44. Torres AD, Rai AN, Hardiek ML (2000) Mercury intoxication and arterial hypertension: report of two patients and review of the literature. Pediatrics 105: E34.

45. Barregård L, Sällsten G, Järvholm B (1990) Mortality and cancer incidence in chloralkali workers exposed to inorganic mercury. Br J Ind Med 47: 99-104.

46. Wojciechowski J, Kowalski W (1975) Cardiac and aortic lesions in chronic experimental poisoning with mercury vapors. Pol Med Sci Hist Bull 15: 255-260.

47. Rissanen T, Voutilainen S, Nyyssonen K, Lakka TA, Salonen JT (2000) Fish oilderived fatty acids, docosahexaenoic acid and docosapentaenoic acid, and the risk of acute coronary events: the Kuopio Ischaemic Heart Disease Risk Factor Study. Circulation 102: 2677-2679.

48. Virtanen JK, Voutilainen S, Rissanen TH, Mursu J, Tuomainen TP, et al (2005) Mercury, fish oils, and risk of acute coronary events and cardiovascular disease, coronary heart disease, and all-cause mortality in men in eastern Finland. Arterioscler Thromb Vasc Biol 25: 228-233.

49. Kobal AB, Flisar Z, Miklavcic V, DizdareviÄł T, Sesek-Briski A (2000) Renal function in miners intermittently exposed to elemental mercury vapour. Arh Hig Rada Toksikol 51: 369-380.
50. Connor WE (2000) Importance of n-3 fatty acids in health and disease. Am J Clin Nutr 71: 171S-5S.

51. Burr ML, Fehily AM, Gilbert JF, Rogers S, Holliday RM, et al. (1989) Effects of changes in fat, fish, and fibre intakes on death and myocardial reinfarction: diet and reinfarction trial (DART). Lancet 2: 757-761.

52. (1999) GISSI-Prevenzione Investigators (Gruppo Italiano per lo Studio della Sopravvivenza nell'Infarto miocardico). Dietary supplementation with n-3 polyunsaturated fatty acids and vitamin $\mathrm{E}$ after myocardial infarction: results of the GISSI-Prevenzione trial. Lancet 354: 447-455.

53. Marckmann P, Grønbaek M (1999) Fish consumption and coronary heart disease mortality. A systematic review of prospective cohort studies. Eur J Clin Nutr 53: 585-590.

54. MacIntosh DL, Williams PL, Hunter DJ, Sampson LA, Morris SC, et al. (1997) Evaluation of a food frequency questionnaire-food composition approach for estimating dietary intake of inorganic arsenic and methylmercury. Cancer Epidemiol Biomarkers Prev 6:1043-1050.

55. Garland M, Morris JS, Rosner BA, Stampfer MJ, Spate VL, et al. (1993) Toenail trace element levels as biomarkers: reproducibility over a 6-year period. Cancer Epidemiol Biomarkers Prev 2: 493-497.

56. Mazerik JN, Mikkilineni H, Kuppusamy VA, Steinhour E, Peltz A, et al. (2007) Mercury activates phospholipase a(2) and induces formation of arachidonic Acid metabolites in vascular endothelial cells. Toxicol Mech Methods 17: 541557

57. Mazerik JN, Hagele T, Sherwani S, Ciapala V, Butler S, et al. (2007) Phospholipase A2 activation regulates cytotoxicity of methylmercury in vascular endothelial cells. Int J Toxicol 26: 553-569.

58. Hagele TJ, Mazerik JN, Gregory A, Kaufman B, Magalang U, et al. (2007) Mercury activates vascular endothelial cell phospholipase D through thiols and oxidative stress. Int J Toxicol 26: 57-69.

59. Peltz A, Sherwani SI, Kotha SR, Mazerik JN, O'Connor Butler ES, et al. (2009) Calcium and calmodulin regulate mercury-induced phospholipase $D$ activation in vascular endothelial cells. Int J Toxicol 28: 190-206.

60. Wiggers GA, Peçanha FM, Briones AM, Pérez-Girón JV, Miguel M, et al. (2008) Low mercury concentrations cause oxidative stress and endothelial dysfunction in conductance and resistance arteries. Am J Physiol Heart Circ Physiol 295: $\mathrm{H} 1033-1033 \mathrm{H} 1043$.

61. Al-Saleh I, Shinwari N, Mashhour A, Mohamed Gel-D, Ghosh MA, et al. (2006) Cadmium and mercury levels in Saudi women and its possible relationship with hypertension. Biol Trace Elem Res 112: 13-29.

62. Fillion M, Mergler D, Sousa Passos CJ, Larribe F, Lemire M, et al. (2006) A preliminary study of mercury exposure and blood pressure in the Brazilian Amazon. Environ Health 5: 29.

63. Bautista LE, Stein JH, Morgan BJ, Stanton N, Young T, et al. (2009) Association of blood and hair mercury with blood pressure and vascular reactivity. WMJ 108: $250-252$.

64. Valera B, Dewailly E, Poirier P (2009) Environmental mercury exposure and blood pressure among Nunavik Inuit adults. Hypertension 54: 981-986.

65. Yorifuji T, Tsuda T, Kashima S, Takao S, Harada M (2010) Long-term exposure to methylmercury and its effects on hypertension in Minamata. Environ Res 110: 40-46.

66. Vupputuri S, Longnecker MP, Daniels JL, Guo X, Sandler DP (2005) Blood mercury level and blood pressure among US women: results from the National Health and Nutrition Examination Survey 1999-2000. Environ Res 97: 195-200.

67. Moszczyski $P$ (2006) Mercury and the risk of coronary heart disease. Przegl Lek 63 Suppl 7: 84-87.

68. Virtanen JK, Rissanen TH, Voutilainen S, Tuomainen TP (2007) Mercury as a risk factor for cardiovascular diseases. J Nutr Biochem 18: 75-85.

69. Wennberg M, Strömberg U, Bergdahl IA, Jansson JH, Kauhanen J, et al. (2012) Myocardial infarction in relation to mercury and fatty acids from fish: a riskbenefit analysis based on pooled Finnish and Swedish data in men. Am J Clin Nutr 96: 706-713.

70. Virtanen JK, Laukkanen JA, Mursu J, Voutilainen S, Tuomainen TP (2012) Serum long-chain $n-3$ polyunsaturated fatty acids, mercury, and risk of sudden cardiac death in men: a prospective population-based study. PLoS One 7 : e41046. 
71. Mozaffarian D, Shi P, Morris JS, Spiegelman D, Grandjean P, et al. (2011) Mercury exposure and risk of cardiovascular disease in two U.S. cohorts. N Engl J Med 364: 1116-1125.

72. Kim DS, Lee EH, Yu SD, Cha JH, Ahn SC (2005) Heavy metal as risk factor of cardiovascular disease--an analysis of blood lead and urinary mercury. J Prev Med Public Health 38: 401-407.

73. Smith KM, Barraj LM, Kantor M, Sahyoun NR (2009) Relationship between fish intake, n-3 fatty acids, mercury and risk markers of $\mathrm{CHD}$ (National Health and Nutrition Examination Survey 1999-2002). Public Health Nutr 12: 1261-1269.

74. Skoczyska A, Jedrejko M, Martynowicz H, Poreba R, Affelska-Jercha A, et al. (2010) The cardiovascular risk in chemical factory workers exposed to mercury vapor. Med Pr 61: 381-391.

75. Wennberg M, Bergdahl IA, Stegmayr B, Hallmans G, Lundh T, et al. (2007) Fish intake, mercury, long-chain n-3 polyunsaturated fatty acids and risk of stroke in northern Sweden. Br J Nutr 98: 1038-1045.
76. Lim KM, Kim S, Kim K, Jang WH, Bae ON, et al. (2010) Low- level mercury can enhance procoagulant activity of erythrocytes: a new contributing factor for mercury-related thrombotic disease. Environ Health Perspect. 118: 928-935.

77. Pedersen EB, Jørgensen ME, Pedersen MB, Siggaard C, Sørensen TB, et al. (2005) Relationship between mercury in blood and 24-h ambulatory blood pressure in Greenlanders and Danes. Am J Hypertens 18: 612-618.

78. García Gómez M, Boffetta P, Caballero Klink JD, Español S, Gómez Quintana $\mathrm{J}$ (2007) Cardiovascular mortality in mercury miners. Med Clin (Barc) 128: 766771

79. Choi AL, Weihe P, Budtz-Jørgensen E, Jørgensen PJ, Salonen JT, et al (2009) Methylmercury exposure and adverse cardiovascular effects in Faroese whaling men. Environ Health Perspect 117: 367-372.

80. Valera B, Dewailly E, Poirier P (2008) Cardiac autonomic activity and blood pressure among Nunavik Inuit adults exposed to environmental mercury: a cross-sectional study. Environ Health 7: 29. 